\title{
A simple and rapid immunochromatography test based on readily available filter paper modified with chitosan to screen for 13 sulfonamides in milk
}

\author{
Yuyang Zeng, 일 Demei Liang, $\odot$ Pimiao Zheng, Yanfang Zhang, Zile Wang, $\odot$ Ghulam Mujtaba Mari, \\ and Haiyang Jiang* (D) \\ Beijing Advanced Innovation Center for Food Nutrition and Human Health, College of Veterinary Medicine, China Agricultural University, \\ Beijing Key Laboratory of Detection Technology for Animal-Derived Food Safety, Beijing Laboratory for Food Quality and Safety, 100193 Beijing, \\ People's Republic of China
}

\section{ABSTRACT}

In this study, we developed a novel, simple, rapid, and low-cost colloidal gold-based immunochromatography method, with filter paper replacing nitrocellulose membrane as the substrate. To obtain adequately immobilized protein, chitosan was used to functionalize the filter paper. After conditions and parameters were optimized, the novel immunochromatography method was applied for detection of sulfonamide residues in milk. Quantitative detection was accomplished using a smartphone and Photoshop software (Adobe Inc., San Jose, CA), allowing us to screen 13 sulfonamides with a limit of detection ranging from 0.42 to $8.64 \mu \mathrm{g} / \mathrm{L}$ and recovery ranging from 88.2 to $116.9 \%$ in milk. The proposed novel method performed similarly to the conventional method that uses a nitrocellulose membrane as the transport medium, and it had lower cost and better usability because of the inexpensive and easily available filter paper.

Key words: filter paper, chitosan, lateral flow device, sulfonamides

\section{INTRODUCTION}

Antibiotics are widely used in dairy farming and milk production to inhibit growth of bacteria and promote the growth of dairy cows (Li et al., 2019; Rana et al., 2019). Sulfonamides (SA), derivatives of $p$-aminobenzenesulfonamide, are one of the most widely used types of antimicrobial drugs because of their high efficiency and cost effectiveness (Baran et al., 2011). They have

Received May 31, 2020.

Accepted August 27, 2020.

*Corresponding author: haiyang@cau.edu.cn broad-spectrum antibacterial properties. They can affect bacterial reproduction by inhibiting bacterial folate metabolism and interfering with synthesis of bacterial nucleic acids and proteins (Dmitrienko et al., 2014). However, their ubiquitous use in veterinary practice leads to residues in foodstuffs and the environment, including milk, meat, and rivers. Furthermore, the human intake of SA residues through foods over time leads to adverse effects, allergies, poisoning, and even cancer (Wang et al., 2017). According to the European Commission, the Food and Drug Administration, and the Chinese Ministry of Agriculture, the maximum residue level of SA is $100 \mu \mathrm{g} / \mathrm{kg}$ in animal foods (European Commission, 1990; Chinese Ministry of Agriculture, 2002; US Food and Drug Administration, 2003). Therefore, the detection of SA residues in milk consumed by the human has become a function for public health agencies.

Colloidal gold immunochromatography (ICA) is an effective and powerful tool for point-of-care food safety testing of because it is rapid, simple, sensitive, and portable. Colloidal gold ICA has been widely applied in the screening of antibiotic residues in dairy products (Chen et al., 2017). Nitrocellulose membranes (NCM) are important elements of and are generally used in ICA devices. Nitrocellulose membranes provide the substrate for the immune response and color development of ICA devices, due to their uniform pore size, smooth surface, and high protein immobilization capability (Apilux et al., 2013). However, their relatively high cost and complex manufacturing processes have led to their manufacture being monopolized by a few companies, which limits usage of NCM in developing and remote areas. Therefore, other affordable and available easily materials, such as paper and cotton thread, have been proposed as alternatives for ICA devices (Teerinen et al., 2014; Jia et al., 2017).

Filter paper (FP) consists of a cellulose fiber web and has high mechanical stability and tensile strength; 
it is a common material used in filtration and separation applications in scientific research. It has received considerable attention in microfluidic platforms because of its abundance, low cost, hydrophilic properties, biocompatibility, and ease of modification (He et al., 2015; Ilacas et al., 2019). Paper-based ELISA is the classic paper analytical device: it retains the sensitivity and specificity of traditional ELISA and has the advantages of rapid processing, low solution use, and cost effectiveness of FP (Wang et al., 2012; Busin et al., 2018; Yang et al., 2018). In addition, FP shows excellent capillary action for transporting liquids without any external equipment and it facilitates mass manufacturing. Based on the above characteristics, we considered using FP as a substitute for NCM in ICA devices. However, FP without modification has poor capacity to adsorb protein, which affects the analytical performance of ICA strips. Hence, it is essential to modify filter paper to improve the adsorption capacity for protein.

Chitosan (CS), a high-molecular-weight linear biopolymer, is obtained from deacetylation of chitin, which is widely abundant on earth. Chitosan is considered a functional material because of its biocompatibility, antibacterial activity, and adsorption properties (Ravi Kumar, 2000). It is a structural polysaccharide with a similar structure to cellulose, although it has better mechanical properties and binding affinities with proteins than cellulose. Hence, it is often used to modify FP or cotton thread (both consisting of cellulose) to enhance the wet strength of substrate, the stability of the hydrophilicity, and the ability to immobilize proteins ( $\mathrm{Li}$ et al., 2018; Ma et al., 2018). In this work, we used CS to modify the test and control lines on FP so that the protein could be firmly bonded to the cellulose surface by electrostatic force, hydrogen bonds, and hydrophobic interactions (Li and Peng, 2015; Lim et al., 2015).

In this study, we established a filter paper-based immunochromatographic assay (FP-ICA) to detect SA residues in milk as model analytes. The FP modified with CS showed excellent ability to bind protein and has additional advantages of cost effectiveness and ease of fabrication compared with NCM. As shown in Figure 1, we used the typical gold nanoparticles (GNP) as the label-conjugated antibodies in FP-ICA to permit visual analysis. In addition, we performed a quantitative analysis by calculating and analyzing the colorimetric ratio of the test and control zones using a smartphone and Photoshop software (Adobe Inc., San Jose, CA). Eventually, to validate the developed FP-ICA, we established a traditional ICA based on nitrocellulose membranes (NCM-ICA) and compared the two devices.

\section{MATERIALS AND METHODS}

\section{Chemicals and Instrumentation}

Sulfonamide antigen $(3.914 \mathrm{mg} / \mathrm{mL})$ and anti-SA antibody $(2.33 \mathrm{mg} / \mathrm{mL})$ were prepared in our laboratory. Goat anti-mouse $\operatorname{IgG}(17 \mathrm{mg} / \mathrm{mL})$ was purchased from Arista Biologicals (Allentown, PA). Chitosan (95\% deacetylation) and BSA were purchased from SigmaAldrich (St. Louis, MO). Thirteen sulfonamides, sulfadimethoxine (SDM), sulfachloropyridazine (SCP), sulfamethoxydiazine (SMD), sulfapyridine (SPY), sulfamonomethoxine (SMM), sulfaquinoxaline (SQX), sulfadiazine (SDZ), sulfaquinoxaline (SIM), sulfaethoxypyridazine (SEP), sulfamethazine (SM2), sulfamethoxypyridazine (SMP), sulfamerazine (SMR), and sulfanitran (SAN), and other standards were provided by the National Institution for Food and Drug (Beijing, China). All solvents and other chemicals were of analytical reagent grade.

Whatman chromatography paper No. 3MM Chr (pure cellulose paper, WC 3MM) and Whatman qualitative paper No. 1 (WQ 1), No. 2 (WQ 2) and No. 4 (WQ 4) were purchased from GE Healthcare (Little Chalfont, UK). Xinxing quantitative paper (XQ) was obtained from Hangzhou Special Paper Co. Ltd. (Hangzhou, China). Scotch tape was purchased from local stationery shop in Beijing, China. The absorbent pad, sample pad, and polyvinyl chloride (PVC) cards were obtained from Kinbio Tech Co. Ltd. (Shanghai, China). Ultrapure water was purified using a Milli-Q system from Millipore Corp. (Bedford, MA). Ultrasonic equipment was supplied by Zhixin Instrument Co. Ltd. (Shanghai, China). An XYZ dispensing platform (HM3035) and programmable strip cutter ZQ4000 were obtained from Kinbio Tech Co. Ltd. (Shanghai, China). The shooting box was acquired from our laboratory.

\section{Synthesis of GNP and GNP-Antibody Conjugates}

Gold nanoparticles with an average diameter of 40 $\mathrm{nm}$ were prepared according to a published method (Hermanson, 2013). Briefly, $1 \mathrm{~mL}$ of $1 \%$ trisodium citrate solution was added rapidly to $50 \mathrm{~mL}$ of boiling $0.02 \% \mathrm{HAuCl}_{4}$ solution with stirring. After the color stabilized, the GNP solution was cooled and stored at room temperature for future immunoassay testing.

The GNP-antibody conjugates were prepared according to a published protocol (Peng et al., 2014). Initially, diluted antibody (containing $0.5 \%$ BSA) was added to $1 \mathrm{~mL}$ of GNP solution (pH 8.0). After 10 min of incubation, $100 \mu \mathrm{L}$ of $10 \%$ BSA (wt/vol) was added to the 
mixture for $10 \mathrm{~min}$ and the mixture was then centrifuged at $8,600 \times g$ for $10 \mathrm{~min}$ at $4^{\circ} \mathrm{C}$. Subsequently, the complex was resuspended in $200 \mu \mathrm{L}$ of PBS (0.01 $M, \mathrm{pH}$ 7.4) containing Tween-20, polyethylene glycol (PEG) 20000, and sucrose. After 3 min of sonication, the conjugates were stored at $4^{\circ} \mathrm{C}$ until use.

\section{Assembling of Strips}

The filter paper was initially coated with $0.5 \mathrm{mg} / \mathrm{mL}$ $\mathrm{CS}(\mathrm{pH}$ 6.5) and dried at room temperature for $15 \mathrm{~min}$. The amino group in CS has a positive charge and binds to proteins with a negative charge under weakly acidic conditions. Then, the antigen and goat anti-mouse IgG $(2.2 \mathrm{mg} / \mathrm{mL})$, respectively, were spotted on the test $(\mathbf{T})$ and control $(\mathbf{C})$ lines to combine CS on the filter paper, with a dispensed volume of $0.5 \mu \mathrm{L} / \mathrm{cm}$. The prepared filter paper was dried at $45^{\circ} \mathrm{C}$ for $2 \mathrm{~h}$. The sample pad was treated with phosphate buffer (0.02 M, pH 7.4) containing $1 \%$ BSA (wt/vol) and $0.5 \%$ polyvinylpyr- rolidone (wt/vol) and then dried at $60^{\circ} \mathrm{C}$ for $2 \mathrm{~h}$. Then, the sample pad, filter paper, absorbent pad, and PVC card with a layer of scotch tape were assembled as an FP-ICA strip. In order to simulate the smooth side of commercial NC membrane, it was necessary to paste a layer of scotch tape on the PVC card of the FPICA strip. The assembly of the NCM-ICA strip was the same as the process for the FP-ICA strip, except that the PVC card did not include a layer of scotch tape because the commercial NC membrane was already smooth. Finally, the assembled strips were cut to a width of $3.5 \mathrm{~mm}$ and stored dry at room temperature.

\section{FP-ICA Testing and Comparison with Traditional NCM-ICA}

All milk samples were obtained from local cattle farms. Initially, milk samples with different concentrations of SDM were diluted 6 times in $0.01 M$ PBS (pH 7.4) and mixed with GNP-antibody conjugates for 3

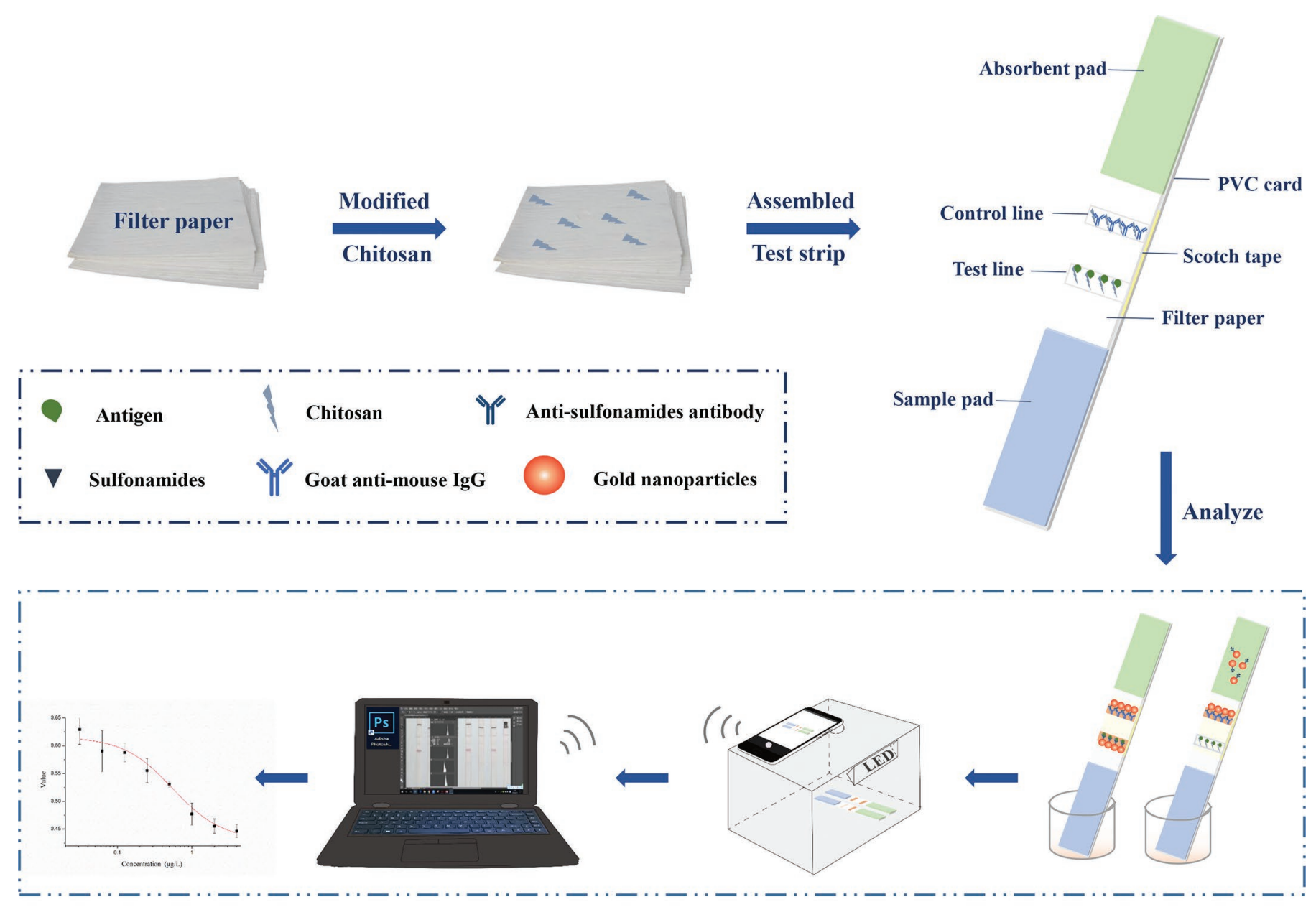

Figure 1. Preparation of colloidal gold test strips with chitosan-functionalized filter paper as substrate used to detect sulfonamides in milk. 
A

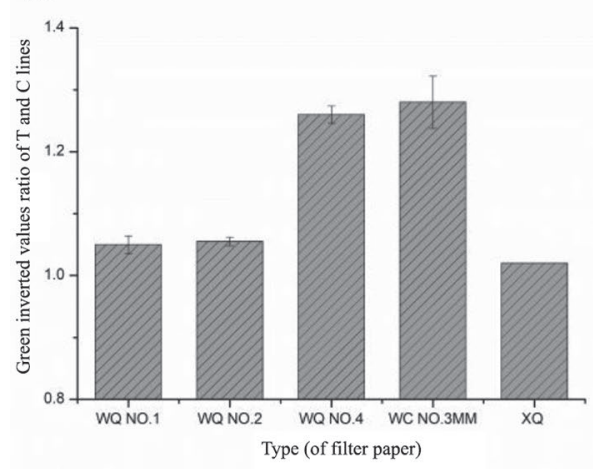

C

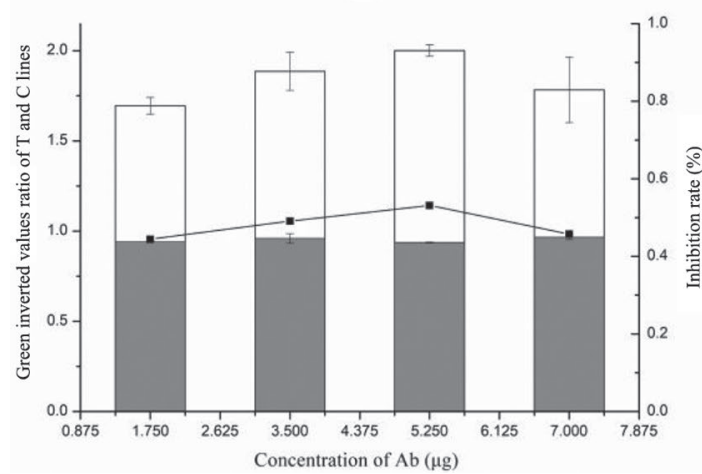

B

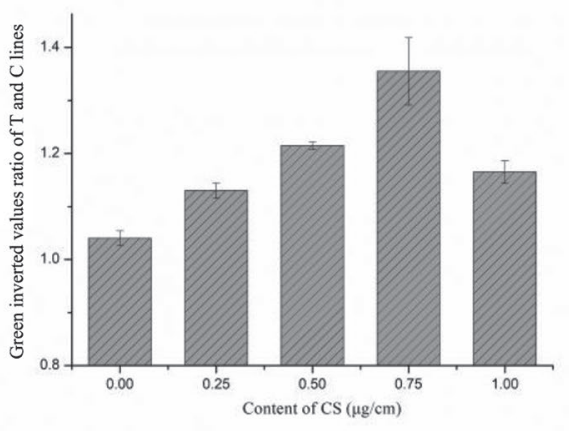

D

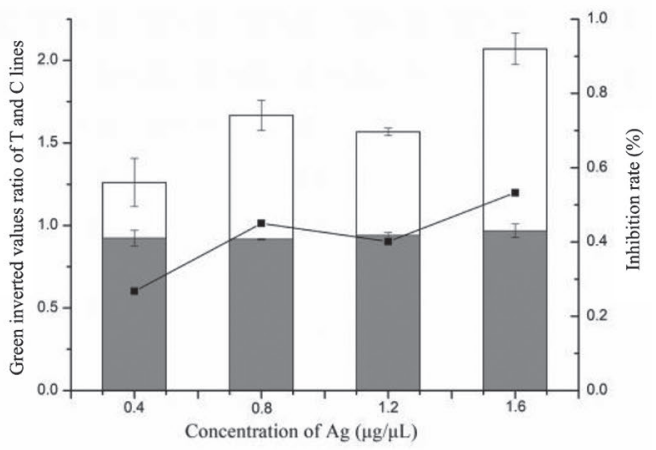

Figure 2. Optimized conditions of filter paper immunochromatography assay (FP-ICA), showing effect of (A) type of filter paper, (B) content of chitosan (CS), (C) concentration of anti-sulfonamide antibody (Ab), and (D) immobilizing concentration of coating antigen (Ag) sulfadimethoxine. Filter papers: WQ No. 1, No. 2, No. 3, No. 4, and WC No. 3MM (pure cellulose paper) were from Whatman, GE Healthcare, Little Chalfont, UK); XQ (Xinxing quantitative paper) was from Hangzhou Special Paper Co. Ltd. (Hangzhou, China). Error bars represent SD.

min. Then, the mixed solution was transmitted to the test strip. After $10 \mathrm{~min}$, the resulting images were captured by smartphone. Finally, the colorimetric results were analyzed using Photoshop software to obtain the green inverted values ratio of the $\mathrm{T}$ and $\mathrm{C}$ lines. All experiments were performed in triplicate. Under the same conditions, traditional NCM-ICA was compared with FP-ICA to demonstrate the feasibility of FP-ICA. The remaining 12 sulfonamides were detected using the same procedure.

\section{Statistical Analysis}

Using OriginPro 8.5 software (https://www.originlab .com), the calibration curve was established by the operation of sigmoidal fit, and the 4-parameter equation was obtained. Then, the analytical performance of sensitivity, specificity, and accuracy for FP-ICA was obtained by the equation. One-way ANOVA method in IBM SPSS Statistics 19 (IBM Corp., Armonk, NY) was used for the stability test of FP-ICA. The stability of the strip was estimated by the $P$-value.

\section{RESULTS AND DISCUSSION}

\section{Optimization of FP-ICA}

Optimization of $\boldsymbol{F P}$. To demonstrate the effect of FP on color intensity, several commonly used filter papers, including WC 3MM, WQ 1, WQ 2, WQ 4, and $\mathrm{XQ}$, were considered in this study. As shown in Figure 2a, FP differing in purity of cellulose and pore size exhibited different color intensities. The WQ 4 with a pore size of $20 \mu \mathrm{m}$ resulted in higher intensity of color compared with other FP with smaller pore size. The WC 3MM FP, which is made of pure cellulose, exhibited the highest color intensity. Therefore, WC 3MM was chosen for further experiments.

Optimization of Chitosan Content. As shown by infrared spectroscopy (Supplemental Figure S1; https: //doi.org/10.3168/jds.2020-18987), chitosan can crosslink with FP. Furthermore, CS has high adsorption to proteins through hydrophobic and electrostatic contacts and slightly inhibits surface hydrophilicity of FP (Bordenave et al., 2007; Orelma et al., 2011; Bekale et al., 2015). Hence, the color intensity band on the $\mathrm{T}$ and 
$\mathrm{C}$ zones is closely related to the CS content. We studied the effect of CS using contents of $0,0.25,0.5,0.75$, and $1 \mu \mathrm{g} / \mathrm{cm}$. As shown in Figure 2b, color intensity increased with increasing CS content up to $0.75 \mu \mathrm{g} / \mathrm{cm}$. However, intensity decreased at CS content $>0.75 \mu \mathrm{g} /$ $\mathrm{cm}$, likely because the greater content of CS resulted in more nonspecific adsorption and increased background. Therefore, we chose a CS content of $0.75 \mu \mathrm{g} / \mathrm{cm}$ for further experiments.

Optimization of Content of Anti-SA Antibody. The use of a suitable concentration of the anti-SA antibody would enhance sensitivity and reduce the cost of FP-ICA. Antibody contents of $1.75,3.5,5.25$, and $7 \mu \mathrm{g}$ were used in the study. As shown in Figure 2c, the results indicated that intensity increased with increasing concentration of anti-SA antibody. However, intensity was weak at an antibody concentration greater than $5.25 \mu \mathrm{g}$ because the background value was enhanced by the increased antibody content. Therefore, an antiSA antibody content of $5.25 \mu \mathrm{g}$ was chosen for further experiments.

Optimization of Concentration of Coating Antigen. After obtaining the optimal concentration of the anti-SA Ab, we needed to establish an appropriate concentration of the coating antigen in the $\mathrm{T}$ zone to improve the color intensity. Antigen concentrations of $0.4,0.8,1.2$, and $1.6 \mu \mathrm{g} / \mu \mathrm{L}$ were used in the study. As shown in Figure 2d, intensity increased with increasing concentration of antigen in general. However, immobilizing antigen at $0.8 \mu \mathrm{g} / \mu \mathrm{L}$ resulted in higher intensity than antigen at $1.2 \mu \mathrm{g} / \mu \mathrm{L}$. Hence, considering cost and color intensity, an antigen concentration of $0.8 \mu \mathrm{g} / \mu \mathrm{L}$ was chosen.

\section{Analytical Performance}

Sensitivity of FP-ICA and NCM-ICA. After defining the optimal conditions, milk samples containing different concentrations of SDM in range of 0 to 24 $\mu \mathrm{g} / \mathrm{L}$ were diluted 6 times using $0.01 M$ PBS (pH 7.4) and detected by FP-ICA and NCM-ICA. Results are shown in Figure 3a and b; cut-off values for FP-ICA and NCM-ICA were 12 and $6 \mu \mathrm{g} / \mathrm{L}$, respectively, for detection by the naked eye. The quantitative analysis using Photoshop is shown in Figure 3c and d. The resulting calibration curves of FP-ICA and NCM-ICA were $\mathrm{Y}=0.916+0.487 /\left[1+(\mathrm{X} / 0.745]^{2.504}\right)$ and $\mathrm{Y}=$ $0.442+0.049 /\left[1+(\mathrm{X} / 0.604)^{1.968}\right]$, where $\mathrm{Y}$ is the green inverted value ratio of the $\mathrm{T}$ line and $\mathrm{C}$ line, and $\mathrm{X}$ is the concentration of SDM); the coefficients of determination $\left(\mathrm{R}^{2}\right)$ were 0.994 and 0.989 , respectively. The linear ranges of FP-ICA and NCM-ICA were from 2.42 to $7.74 \mu \mathrm{g} / \mathrm{L}$ and from 1.80 to $7.32 \mu \mathrm{g} / \mathrm{L}$, respectively.
The limit of detection (LOD) was estimated as 10\% inhibition $\left(\mathrm{IC}_{10}\right)$ of the standard curve (Sun et al., 2017). Hence, the LOD for FP-ICA and NCM-ICA were 1.86 and $1.20 \mu \mathrm{g} / \mathrm{L}$ in milk, respectively.

Specificity of FP-ICA. The specificity of FPICA was estimated in a cross-reactivity test with 30 other antibacterial agents. The results are shown in Supplemental Table S1 and Supplemental Figure S2 (https://doi.org/10.3168/jds.2020-18987). The anti-SA antibody reacted negligibly with drugs other than the 12 sulfonamides (SCP, SMD, SPY, SMM, SQX, SDZ, SIM, SEP, $\mathrm{SM}_{2}$, SMP, SMR, and SAN). Therefore, we can consider this antibody to have broad-spectrum activity against sulfonamides. The FP-ICA established in this study could simultaneously detect $13 \mathrm{SA}$ in milk samples (Table 1), with LOD ranging from 0.42 to 8.64 $\mu \mathrm{g} / \mathrm{L}$.

Accuracy and Precision of FP-ICA. To confirm the practical applicability of the FP-ICA, recovery was tested in milk samples spiked with different concentration of sulfonamides. Five of the 13 SA (SMD, SAN, $\mathrm{SDZ}, \mathrm{SMM}$, and $\mathrm{SM}_{2}$ ) were tested and results are shown in Table 2. The average recoveries for SA in milk ranged from 88.2 to $116.9 \%$, with a relative standard deviation (RSD) $<18 \%$.

Stability of FP-ICA. We tested the stability of the FP strip using the Arrhenius equation $\left(k=A T^{m} e^{-\frac{E_{0}}{R T}}\right)$, where $k=$ specific reaction rate; $A T^{m}=$ preexponential factor, $E_{0}=$ apparent activation energy; $R=$ gas constant; and $T=$ thermodynamic temperature, by placing the strips at $55^{\circ} \mathrm{C}$ for 2 consecutive weeks (Wang et al., 2013). The intensity values were almost constant, as shown in Supplemental Figure S3 (https://doi.org/ 10.3168/jds.2020-18987). According to one-way ANOVA, the homogeneity of variance was $P=0.659$, and the single factor analysis resulted in $P=0.409$, indicating no decline in intensity values from 0 to $14 \mathrm{~d}$. This means the strip could be stored stably for 1 yr at room temperature.

Method Validation. To validate the developed FPICA, we tested 10 milk samples that were negative for sulfonamides, 3 of which were randomly spiked with a certain concentration of SDM, confirmed by instrument, and detected using the developed FP-ICA and NCM-ICA. The results showed that both methods had good consistency (Table 3). The performance of the developed FP-ICA was similar to that of the conventional NCM-ICA, but the cost was lower and the preparation process was simpler for FP than NCM. The cost of $\mathrm{FP}$ is about $10 \%$ of the cost of NCM for similar lengths, and it is easier to obtain and use than NCM. 
A

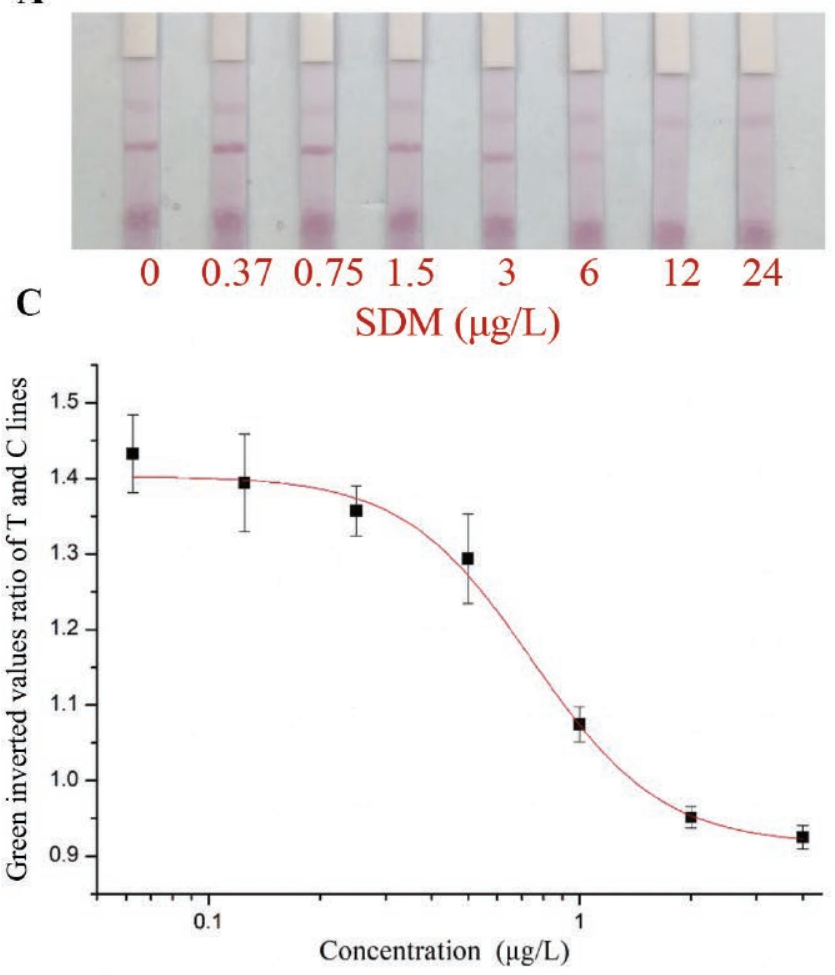

B
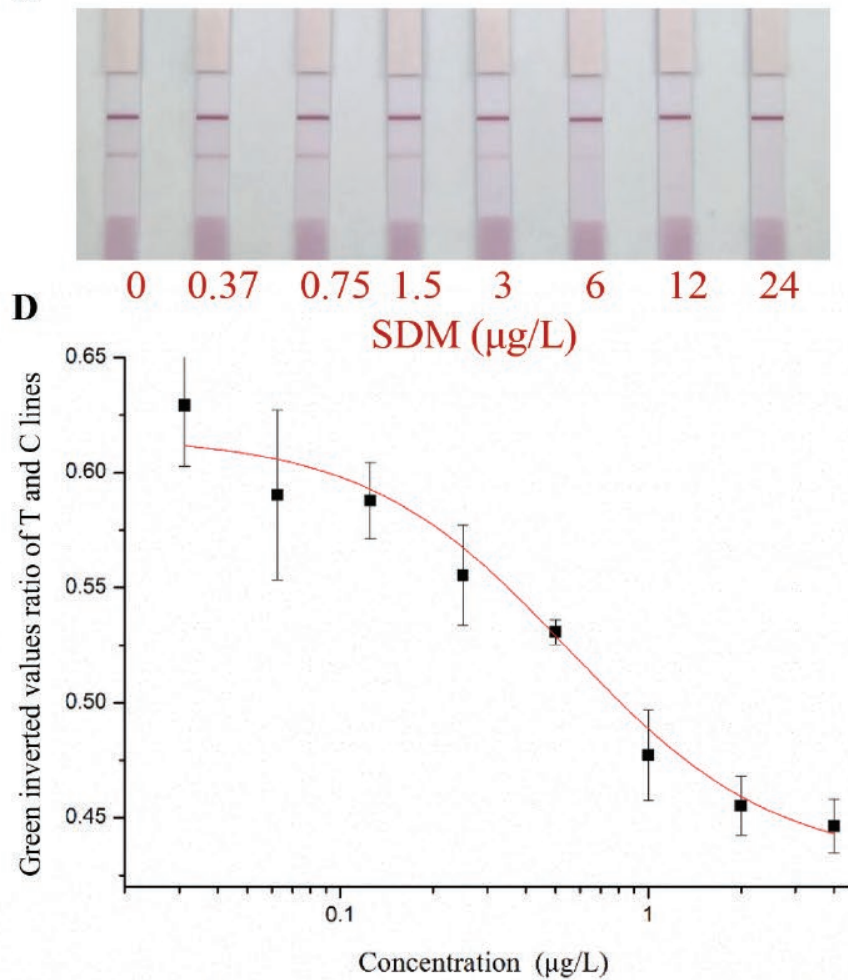

Figure 3. Appearance of test strips and calibration curves of filter paper immunochromatography assay (FP-ICA) and nitrocellulose membrane immunochromatography assay (NCM-ICA) for detection of sulfadimethoxine (SDM) in milk. (A) FP-ICA and (B) NCM-ICA at different concentrations of SDM; calibration curve of (C) FP-ICA and (D) NCM-ICA. Error bars represent SD.

Therefore, the developed FP-ICA uses readily available components and is practical. The method is based on a broad-spectrum antibody, and its purpose is to prevent missed detection of sulfonamides to the extent possible. After samples positive for sulfonamides are detected, the type and residual amount of the sulfonamide need to be verified using an instrument method.

\section{CONCLUSIONS}

The proposed FP-ICA using filter paper functionalized with chitosan as an analytical membrane exhibited good performance for detecting SA residues in milk, sufficient to meet testing standards of legal limits. The chitosan-modified filter paper greatly enhanced the

Table 1. Cross-reactivity of sulfonamide $\mathrm{mAb}$ with different sulfonamides

\begin{tabular}{|c|c|c|c|c|}
\hline Sulfonamide & $\begin{array}{l}\text { Adjusted } \\
\mathrm{R}^{2}\end{array}$ & $\begin{array}{l}\mathrm{LOD}^{1} \\
(\mu \mathrm{g} / \mathrm{L})\end{array}$ & $\begin{array}{l}\text { Linear range } \\
\qquad(\mu \mathrm{g} / \mathrm{L})\end{array}$ & $\begin{array}{l}\text { Cross- } \\
\text { reactivity }(\%)\end{array}$ \\
\hline Sulfadimethoxine & 0.994 & 1.86 & $2.42-7.74$ & 100 \\
\hline Sulfachloropyridazine & 0.979 & 2.94 & $3.72-12.30$ & 65 \\
\hline Sulfamethoxydiazine & 0.999 & 2.88 & $3.42-10.80$ & 73 \\
\hline Sulfapyridine & 0.992 & 3.06 & $4.02-9.72$ & 71 \\
\hline Sulfamonomethoxine & 0.999 & 2.40 & $3.96-22.20$ & 47 \\
\hline Sulfaquinoxaline & 0.996 & 3.00 & $6.18-73.80$ & 21 \\
\hline Sulfadiazine & 0.953 & 8.64 & $14.94-97.10$ & 12 \\
\hline Sulfaquinoxaline & 0.988 & 1.11 & $2.22-24.42$ & 60 \\
\hline Sulfaethoxypyridazine & 0.991 & 0.42 & $1.02-20.40$ & 97 \\
\hline Sulfamethazine & 0.997 & 1.26 & $2.28-15.60$ & 75 \\
\hline Sulfamethoxypyridazine & 0.987 & 1.74 & $2.82-15.06$ & 68 \\
\hline Sulfamerazine & 0.960 & 1.38 & $2.52-18.84$ & 65 \\
\hline Sulfanitran & 0.995 & 1.44 & $2.16-8.88$ & 101 \\
\hline
\end{tabular}

${ }^{1}$ Limit of detection. 
Table 2. Recovery of sulfonamides in spiked milk samples by filter paper immunochromatography assay (FPICA)

\begin{tabular}{|c|c|c|c|c|}
\hline Sulfonamide & $\begin{array}{l}\text { Spiked } \\
(\mu \mathrm{g} / \mathrm{L})\end{array}$ & $\begin{array}{c}\text { Measured } \\
(\mu \mathrm{g} / \mathrm{L})\end{array}$ & $\begin{array}{c}\text { Recovery } \\
(\%)\end{array}$ & $\begin{array}{c}\mathrm{RSD}^{1} \\
(\%)\end{array}$ \\
\hline \multirow[t]{3}{*}{ Sulfamethoxydiazine } & 2.4 & $2.49 \pm 0.41$ & 103.9 & 16.4 \\
\hline & 4.8 & $4.41 \pm 0.40$ & 91.8 & 8.9 \\
\hline & 7.2 & $6.50 \pm 1.14$ & 90.3 & 17.6 \\
\hline \multirow[t]{3}{*}{ Sulfanitran } & 2.4 & $2.58 \pm 0.22$ & 107.8 & 6.8 \\
\hline & 4.8 & $4.95 \pm 0.54$ & 103.2 & 10.7 \\
\hline & 8.4 & $7.79 \pm 1.26$ & 92.7 & 16.6 \\
\hline \multirow[t]{3}{*}{ Sulfadiazine } & 21 & $19.70 \pm 1.44$ & 109.5 & 7.4 \\
\hline & 54 & $54.12 \pm 7.38$ & 100.2 & 13.6 \\
\hline & 80 & $88.92 \pm 12.36$ & 98.8 & 13.9 \\
\hline \multirow[t]{3}{*}{ Sulfamonomethoxine } & 4.8 & $4.50 \pm 0.37$ & 93.5 & 8.2 \\
\hline & 8 & $9.12 \pm 0.48$ & 101.1 & 5.6 \\
\hline & 18 & $20.58 \pm 3.18$ & 114.2 & 15.6 \\
\hline \multirow[t]{3}{*}{ Sulfamethazine } & 3 & $3.48 \pm 0.53$ & 116.9 & 15.1 \\
\hline & 8 & $7.93 \pm 0.43$ & 88.2 & 5.4 \\
\hline & 15 & $15.90 \pm 2.29$ & 106 & 14.4 \\
\hline
\end{tabular}

${ }^{1}$ Relative standard deviation.

ability to immobilize proteins by electrostatic force, hydrogen bonds, and hydrophobic interactions. Thus, FP can be used as a substitute for conventional NCM in ICA. Compared with NCM-ICA, the FP-ICA strip is cheaper and more readily available. Hence, this cost-effective, simple, and rapid paper-based analytical device has potential for use in residue determination, especially in remote areas and low-resource regions. Further studies will focus on detection of other analytes in different samples and using different materials to functionalize the filter paper.

\section{ACKNOWLEDGMENTS}

We thank Tao Peng, Sanlei Xie, Jianyi Wang, and Wenbo Yu (Beijing Key Laboratory of Detection Technology for Animal-Derived Food Safety, Beijing Laboratory for Food Quality and Safety, Beijing, China)

Table 3. Validation assessment $(10$ milk samples with negative sulfonamides, 3 of which were randomly spiked with a certain concentration of sulfadimethoxine) of filter paper immunochromatography assay (FP-ICA)

\begin{tabular}{lccc}
\hline & \multirow{2}{*}{$\begin{array}{c}\text { Spiked } \\
(\mu \mathrm{g} / \mathrm{L})\end{array}$} & FP-ICA strip & NCM-ICA $^{1}$ strip \\
\cline { 2 - 4 } Sample & - & - & - \\
\hline 1 & - & - & - \\
2 & - & $3.21 \pm 0.21$ & $2.73 \pm 0.38$ \\
3 & 3.0 & $4.76 \pm 0.32$ & $4.20 \pm 0.26$ \\
4 & 5.0 & - & - \\
5 & - & $-13 \pm 1.02$ & $6.64 \pm 1.16$ \\
6 & - & - & - \\
7 & 7.0 & - & - \\
9 & - & & - \\
\hline
\end{tabular}

${ }^{1}$ Nitrocellulose membrane immunochromatography. for their guidance in this experiment. This work was financially supported by the Ministry of Science and Technology of the People's Republic of China (Beijing, China; 2015BAK36B03). The authors have not stated any conflicts of interest.

\section{REFERENCES}

Apilux, A., Y. Ukita, M. Chikae, O. Chailapakul, and Y. Takamura. 2013. Development of automated paper-based devices for sequential multistep sandwich enzyme-linked immunosorbent assays using inkjet printing. Lab Chip 13:126-135. https://doi.org/10.1039/ C2LC40690J.

Baran, W., E. Adamek, J. Ziemiańska, and A. Sobczak. 2011. Effects of the presence of sulfonamides in the environment and their influence on human health. J. Hazard. Mater. 196:1-15. https://doi .org/10.1016/j.jhazmat.2011.08.082.

Bekale, L., D. Agudelo, and H. A. Tajmir-Riahi. 2015. Effect of polymer molecular weight on chitosan-protein interaction. Colloids Surf. B Biointerfaces 125:309-317. https://doi.org/10.1016/j .colsurfb.2014.11.037.

Bordenave, N., S. Grelier, F. Pichavant, and V. Coma. 2007. Water and moisture susceptibility of chitosan and paper-based materials: Structure-property relationships. J. Agric. Food Chem. 55:94799488. https://doi.org/10.1021/jf070595i.

Busin, V., S. Burgess, and W. Shu. 2018. A hybrid paper-based microfluidic platform toward veterinary P-ELISA. Sens. Actuators B Chem. 273:536-542. https://doi.org/10.1016/j.snb.2018.06.075.

Chen, Y., L. Liu, Z. Xie, J. Zhu, S. Song, and H. Kuang. 2017. Gold immunochromatographic assay for trimethoprim in milk and honey samples based on a heterogenous monoclonal antibody. Food Agric. Immunol. 28:1046-1057. https://doi.org/10.1080/09540105 .2017 .1325843 .

Chinese Ministry of Agriculture. 2002. Establishment of maximum residue levels of veterinary medical products in foodstuffs of animal origin. Regulation No. 235. Ministry of Agriculture, Beijing, China.

Dmitrienko, S. G., E. V. Kochuk, V. V. Apyari, V. V. Tolmacheva, and Y. A. Zolotov. 2014. Recent advances in sample preparation techniques and methods of sulfonamides detection - A review. Anal Chim. Acta 850:6-25. https://doi.org/10.1016/j.aca.2014.08.023.

European Commission. 1990. Establishment of maximum residue levels of veterinary medical products in foodstuffs of animal origin: Council Regulation (EEC) No. 2377/90, Off. J. L224/1. 
He, Y., Y. Wu, J.-Z. Fu, and W.-B. Wu. 2015. Fabrication of paper-based microfluidic analysis devices: A review. RSC Advances 5:78109-78127. https://doi.org/10.1039/C5RA09188H.

Hermanson, G. T. 2013. Bioconjugate Techniques. 3rd ed. Academic Press/Elsevier, Amsterdam, the Netherlands.

Ilacas, G. C., A. Basa, K. J. Nelms, J. D. Sosa, Y. Liu, and F. A. Gomez. 2019. Paper-based microfluidic devices for glucose assays employing a metal-organic framework (MOF). Anal. Chim. Acta 1055:74-80. https://doi.org/10.1016/j.aca.2019.01.009.

Jia, X., T. Song, Y. Liu, L. Meng, and X. Mao. 2017. An immunochromatographic assay for carcinoembryonic antigen on cotton thread using a composite of carbon nanotubes and gold nanoparticles as reporters. Anal. Chim. Acta 969:57-62. https://doi.org/10.1016/j aca.2017.02.040.

Li, H., and L. Peng. 2015. Antimicrobial and antioxidant surface modification of cellulose fibers using layer-by-layer deposition of chitosan and lignosulfonates. Carbohydr. Polym. 124:35-42. https://doi .org/10.1016/j.carbpol.2015.01.071.

Li, J. H., M. H. Yousif, Z. Q. Li, Z. H. Wu, S. L. Li, H. J. Yang, Y. J. Wang, and Z. J. Cao. 2019. Effects of antibiotic residues in milk on growth, ruminal fermentation, and microbial community of preweaning dairy calves. J. Dairy Sci. 102:2298-2307. https:// doi.org/10.3168/jds.2018-15506.

Li, Y. D., W. Y. Li, H. H. Chai, C. Fang, Y. J. Kang, C. M. Li, and L. Yu. 2018. Chitosan functionalization to prolong stable hydrophilicity of cotton thread for thread-based analytical device application. Cellulose 25:4831-4840. https://doi.org/10.1007/s10570-018-1891 -3 .

Lim, C., D. W. Lee, J. N. Israelachvili, Y. Jho, and D. S. Hwang. 2015. Contact time- and pH-dependent adhesion and cohesion of low molecular weight chitosan coated surfaces. Carbohydr. Polym. 117:887-894. https://doi.org/10.1016/j.carbpol.2014.10.033.

Ma, L., A. Nilghaz, J. R. Choi, X. Liu, and X. Lu. 2018. Rapid detection of clenbuterol in milk using microfluidic paper-based ELISA. Food Chem. 246:437-441. https://doi.org/10.1016/j.foodchem .2017.12.022.

Orelma, H., I. Filpponen, L.-S. Johansson, J. Laine, and O. J. Rojas. 2011. Modification of cellulose films by adsorption of CMC and chitosan for controlled attachment of biomolecules. Biomacromolecules 12:4311-4318. https://doi.org/10.1021/bm201236a.

Peng, T., F. S. Zhang, W. C. Yang, D. X. Li, Y. Chen, Y. H. Xiong, H. Wei, and W. H. Lai. 2014. Lateral-flow assay for rapid quantitative detection of clorprenaline residue in swine urine. J. Food Prot. 77:1824-1829. https://doi.org/10.4315/0362-028X.JFP-14-103.

Rana, M. S., S. Y. Lee, H. J. Kang, and S. J. Hur. 2019. Reducing veterinary drug residues in animal products: A review. Food Sci.
Anim. Resour. 39:687-703. https://doi.org/10.5851/kosfa.2019 .665 .

Ravi Kumar, M. N. V. 2000. A review of chitin and chitosan applications. React. Funct. Polym. 46:1-27. https://doi.org/10.1016/ S1381-5148(00)00038-9.

Sun, Y., J. Xie, T. Peng, J. Wang, S. Xie, K. Yao, C. Wang, S. Sun, X. Xia, and H. Jiang. 2017. A new method based on time-resolved fluoroimmunoassay for the detection of streptomycin in milk. Food Anal. Methods 10:2262-2269. https://doi.org/10.1007/s12161-017 $-0797-2$.

Teerinen, T., T. Lappalainen, and T. Erho. 2014. A paper-based lateral flow assay for morphine. Anal. Bioanal. Chem. 406:5955-5965. https://doi.org/10.1007/s00216-014-8001-7.

US Food and Drug Administration. 2003. New guidance for industry on antimicrobial drugs for food animals. US Food and Drug Administration, Washington, DC.

Wang, D.-B., B. Tian, Z.-P. Zhang, J.-Y. Deng, Z.-Q. Cui, R.-F. Yang, X.-Y. Wang, H.-P. Wei, and X.-E. Zhang. 2013. Rapid detection of Bacillus anthracis spores using a super-paramagnetic lateral-flow immunological detection system. Biosens. Bioelectron. 42:661-667. https://doi.org/10.1016/j.bios.2012.10.088.

Wang, H., L. Ren, X. Yu, J. Hu, Y. Chen, G. He, and Q. Jiang. 2017. Antibiotic residues in meat, milk and aquatic products in Shanghai and human exposure assessment. Food Control 80:217-225. https://doi.org/10.1016/j.foodcont.2017.04.034.

Wang, S., L. Ge, X. Song, J. Yu, S. Ge, J. Huang, and F. Zeng. 2012. Paper-based chemiluminescence ELISA: Lab-on-paper based on chitosan modified paper device and wax-screen-printing. Biosens. Bioelectron. 31:212-218. https://doi.org/10.1016/j.bios.2011.10 .019 .

Yang, C.-H., C.-A. Chen, and C.-F. Chen. 2018. Surface-modified cellulose paper and its application in infectious disease diagnosis. Sens. Actuators B Chem. 265:506-513. https://doi.org/10.1016/ j.snb.2018.03.092

\section{ORCIDS}

Yuyang Zeng $\odot$ https://orcid.org/0000-0002-7707-0416 Demei Liang (ํ) https://orcid.org/0000-0002-2899-5113

Zile Wang ๑ https://orcid.org/0000-0002-4513-688X

Haiyang Jiang @ https://orcid.org/0000-0002-0529-0719 